\title{
SIMULATION OF POWDER SINTERING USING A DISCRETE ELEMENT MODEL
}

\author{
Jerzy ROJEK*, Szymon NOSEWICZ, Katarzyna PIETRZAK",*; Marcin CHMIELEWSKI ${ }^{* *}$ \\ *Institute of Fundamental Technological Research, Polish Academy of Sciences, ul. Pawińskiego 5B, 02-106 Warsaw, Poland \\ *Institute of Electronic Materials Technology, ul. Wólczyńska 133, 01-919 Warsaw, Poland \\ jrojek@ippt.gov.pl, snosew@ippt.gov.pl, katarzyna.pietrzak@itme.edu.pl, chmielem@poczta.fm
}

\begin{abstract}
This paper presents numerical simulation of powder sintering. The numerical model introduced in this work employs the discrete element method which assumes that material can be modelled by a large assembly of discrete elements (particles) of spherical shape interacting among one another. Modelling of sintering requires introduction of the cohesive interaction among particles representing interparticle sintering forces. Numerical studies of sintering have been combined with experimental studies which provided data for calibration and validation of the model. In the laboratory tests evolution of microstructure and density during sintering have been studied. Comparison of numerical and experimental results shows a good performance of the numerical model developed.
\end{abstract}

Key words: Powder Sintering, Simulation, Discrete Element Method

\section{INTRODUCTION}

Sintering is a manufacturing process used for making various parts from metallic or ceramic powder mixtures. Sintering consists in consolidation of loose or weakly bonded powders at elevated temperatures, close to the melting temperature with or without additional pressure. This is a complex process affected by many factors. Numerical simulation of sintering can be used to optimize and to understand better the process and improve the quality of sintered components.

Modelling of sintering process is still a challenging research task. There are different approaches in modeling of sintering processes, ranging from continuum phenomenological models (Abouaf et al., 1988; Duva and Crow, 1992; Cocks, 1989; Sofronis and McMeeking, 1992; Ponte Castañeda, 1991) to atomistic ones (Zeng et al., 1998; Zachariah and Carrier, 1999; Zhu and Averback, 1995; Huilong and Averback, 1996; Matsubara, 1999; Kadau et al., 2002). Growing capabilities of computational techniques increased the possibilities to employ particle sintering models. Sintering models have been implemented within the discrete element method which allows us to model interaction of large collections of particles (Parkami and McMeeking, 1998; Martin et al., 2006; Luding et al., 2005; Olmos et al., 2009; Kadushnikov et al., 2001; Henrich , 2007; Henrich et al., 2007; Wonisch et al., 2009). In the paper of the Parhami and McMeeking (1998) it have been implemented the particle sintering model derived by Coble (1958) in the quasi-static formulation of the lattice type discrete element method to study free and pressureassisted sintering. The concepts of Parhami and McMeeking have been incorporated in the dynamic formulation of the discrete element method by Martin et al. (2006) and used for investigation of free sintering of metallic powders. A similar model has been applied by Henrich et al. (2007) to simulate the free and pressure-assisted solid state sintering of powders with special attention to the grain rearrangement during sintering. The effect of particle size distributions on sintering has been studied by Wonisch et al. (2009).

In the presented work the discrete element method is adopt- ed as a modelling tool. The discrete model has been performed to predict micro- and macroscopic changes during sintering process. The discrete model allows to determine interaction of grains and evaluate the density of material. The numerical model is validated using the results of experimental studies of a sintering process.

\section{EXPERIEMENTAL STUDIES OF A SINTERING PROCESS}

Experimental studies of sintering have been performed in the laboratory of Institute of Electronic Materials Technology. Sintering has been carried out in a Thermal Technology Astro uniaxial hot press shown in Fig. 1. Morphology of the NiAl powder used for sintering is presented in Fig. 2. The samples of the sintered material are shown in Fig. 3. Sintering has been performed under pressure of $30 \mathrm{MPa}$ and at temperature of $1400{ }^{\circ} \mathrm{C}$. Temperature variation during the process is plotted in Fig. 4 . The process was interrupted at different time instants in order to study the evolution of microstructure and kinetics of sintering. The measurement points are marked on the plot in Fig. 4. The kinetics of sintering can be evaluated by investigation of the bulk density change in time. The macroscopic shrinkage of the porous material during sintering leads to the increase of the bulk density. The evolution of the bulk density obtained in our studies is given in Tab. 1. The value of the bulk density close to the theoretical density has been obtained. It means that the material with very low porosity has been obtained by keeping the sample at sintering temperature for a sufficiently long time. The evolution of the bulk density obtained in our studies will be used in calibration of the numerical model.

During sintering particulate material is converted into polycrystal. In the initial stage cohesive bonds (necks) are formed between grains. Microstructure at an early stage of sintering is shown in Fig. 5a. When the sintering process is continued the necks between particle grow (Fig. 5b). Grain rearrangement and increase of grain compaction can be observed during sintering. With the advancement of the sintering process, gradual reduction and elimination of porosity is observed (Figs. $5 b$ and $5 c$ ). 


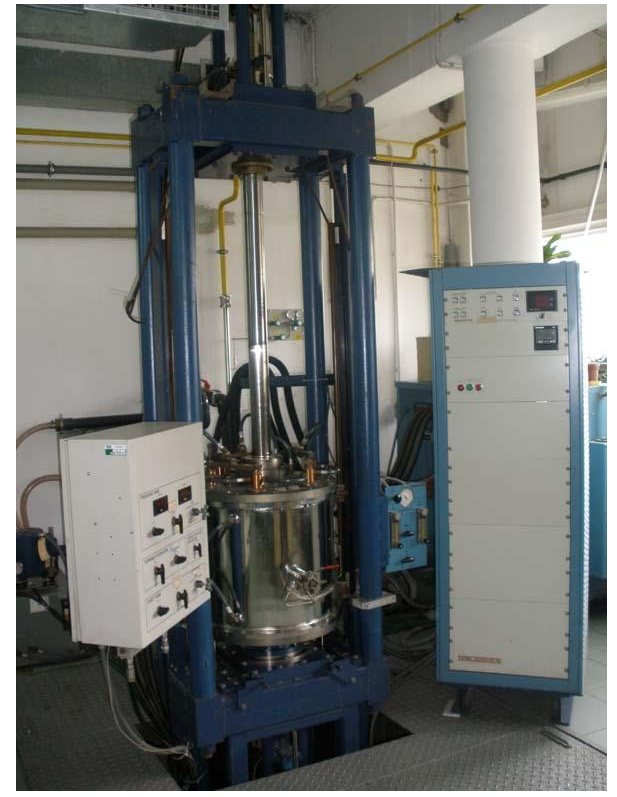

Fig. 1. Thermal Technology Astro uniaxial hot press

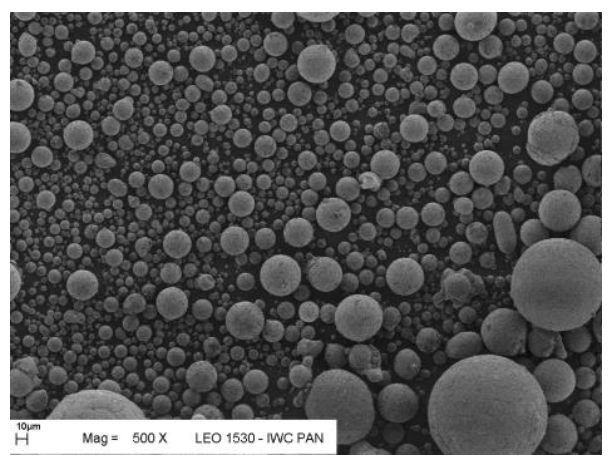

Fig. 2. Morphology of the NiAl powder

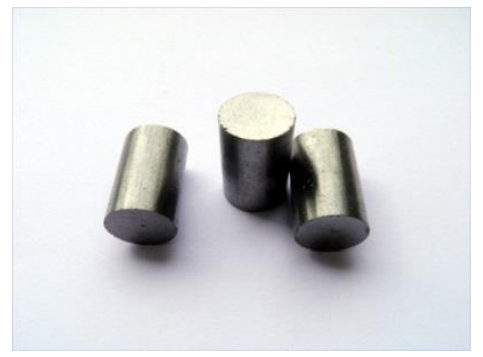

Fig. 3. Samples of sintered material

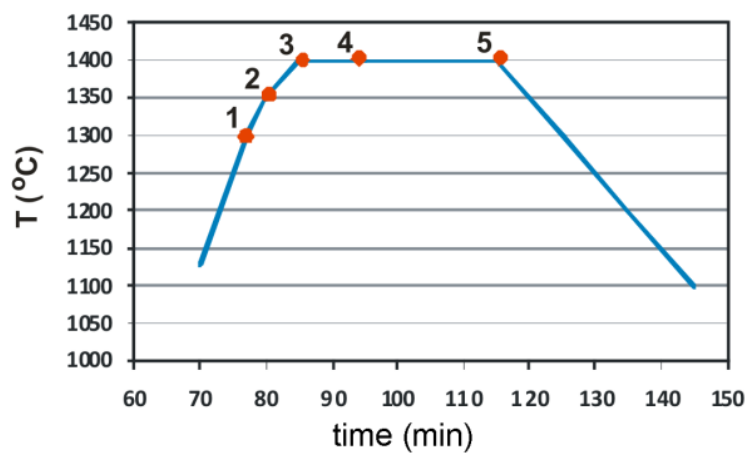

Fig. 4. Temperature variation during sintering of NiAl with measurement points a)

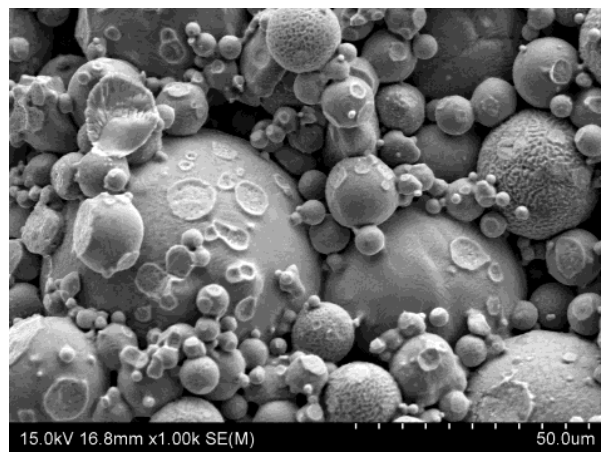

b)

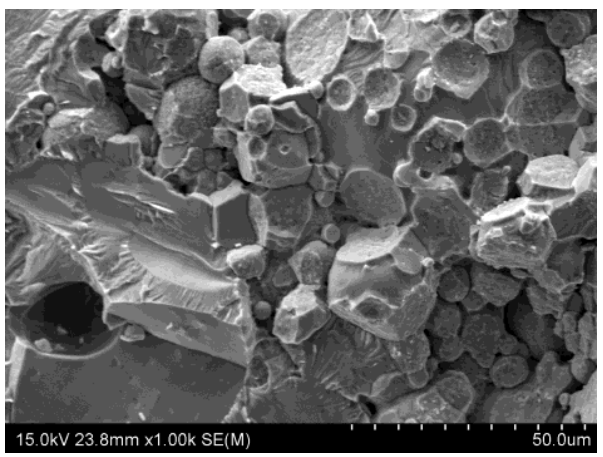

c)

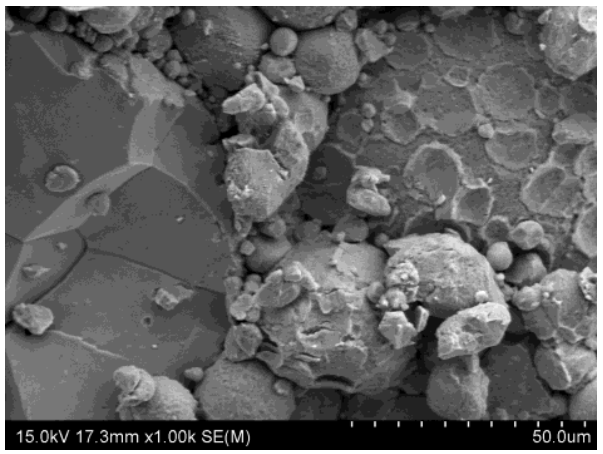

Fig. 5. Microstructure evolution during sintering of NiAl: a) early stage (measurement point P1), b) intermediate stage (P4), b) final stage (P5)

Tab. 1. Density evolution of NiAl during sintering (theoretical density of NiAl: $5.91 \mathrm{~g} / \mathrm{cm}^{3}$ )

\begin{tabular}{|c|c|c|}
\hline $\begin{array}{c}\text { Measurement } \\
\text { point }\end{array}$ & $\begin{array}{c}\text { bulk density } \\
(\mathbf{g} / \mathbf{c m} \mathbf{3})\end{array}$ & $\begin{array}{c}\text { relative density } \\
(\mathbf{\%})\end{array}$ \\
\hline 1 & 5.25 & 88.8 \\
\hline 2 & 5.35 & 90.5 \\
\hline 3 & 5.42 & 91.7 \\
\hline 4 & 5.78 & 97.8 \\
\hline 5 & 5.86 & 99.1 \\
\hline
\end{tabular}

\section{NUMERICAL MODEL OF SINTERING}

Numerical model of sintering is developed within the framework of the discrete element method which assumes that a particulate material can be represented as a collection of spherical particles interacting mutually, thus the discrete element model takes explicitly into account the particulate nature of the sintered material (Rojek et al., 2011). The numerical model of sintering has been implemented in the finite/discrete element code DEMPack (Rojek et al., 2011). 


\subsection{Discrete element method formulation}

In the discrete element method, the translational and rotational motion of rigid spherical elements (particles) is governed by the standard equations of rigid body dynamics. For the $i$-th spherical particle we have:

$$
\begin{aligned}
m_{i} \ddot{\mathbf{u}}_{i} & =\mathbf{F}_{i} \\
J_{i} \dot{\boldsymbol{\omega}}_{i} & =\mathbf{T}_{i}
\end{aligned}
$$

where $\mathbf{u}_{i}$ is the element centroid displacement in a fixed (inertial) coordinate frame $\mathbf{X}, \boldsymbol{\omega}_{i}$ - the angular velocity, $m_{i}$ - the element mass, $J_{i}$ - the moment of inertia, $\mathbf{F}_{i}$ - the resultant force, and $\mathbf{T}_{i}$ - the resultant moment about the central axes. Equations (1) and (2) are integrated in time using the explicit central difference time integration scheme. Taking appropriate contact model we can obtain desired macroscopic behaviour. Therefore the contact model in the discrete element method can be considered as a micromechanical material model. Modelling of sintering requires special model of contact interaction. Below the contact models for powder compaction and powder sintering will be presented.

\subsection{Contact interaction model for powder compaction}

Powder compaction prior to sintering is modelled using the cohesionless contact model with friction. The rheological scheme of this model is shown in Fig. 6. The normal contact interaction is represented by the Kelvin-Voigt element consisting of a spring and a dashpot connected in parallel. The normal contact force $F_{n}$ is a sum of the elastic force in the spring $F_{e}$ and the viscous component $F_{d}$

$$
F_{n}=F_{e}+F_{d}
$$

The elastic part of the normal contact force $F_{e}$ can be evaluated assuming a linear force-displacement relationship:

$$
F_{e}=k_{n} u_{n}
$$

where $k_{n}$ is the normal contact stiffness and $u_{n}$ is the penetration of the two particles, calculated as:

$$
u_{n}=d_{i j}-R_{i}-R_{j}
$$

where $d_{i j}$ is the distance of the particle centres, and $R_{i}$ and $R_{j}$ their radii.

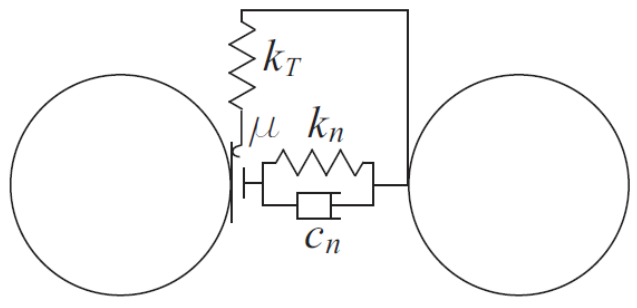

Fig. 6. Rheological scheme of the contact interaction for powder compaction

No cohesion is allowed, so no tensile normal contact forces are allowed:

$$
F_{e} \leq 0
$$

The viscous component of the normal force is assumed to be a linear function of the normal relative velocity $v_{n}$ at the contact point:

$$
F_{d}=c_{n} v_{n}
$$

The value of the viscosity coefficient $c_{n}$ can be taken as a fraction $\xi$ of the critical damping $C_{c r}$ for the system of two rigid bodies with masses $m_{i}$ and $m_{j}$, connected with a spring of the stiffness $k_{n}$ :

$c_{n}=\xi C_{c r}$

where the critical damping can be calculated:

$$
C_{c r}=2 \sqrt{\frac{m_{i} m_{j} k_{n}}{m_{i}+m_{j}}}
$$

The tangential reaction $F_{t}$ at the contact point is brought about by the friction opposing the relative motion. In our model, friction is modelled using the regularized Coulomb friction model characterized by the tangential contact stiffness $k_{t}$ and friction coefficient $\mu$.

\subsection{Contact interaction model for sintering}

When during heating the sintering temperature is reached the interaction model type is switched from that representing powder compaction to the model appropriate for sintering. Conversely, when the temperature drops below the sintering temperature during cooling or when the sintering process reaches equilibrium, the interaction model is switched back to the model presented in Sec. 3.2.

Modelling of sintering requires introduction of a cohesive interaction among particles representing inter-particle sintering forces. As a result of the stresses in the neck and the surface tension the particles are attracted to each other leading to shrinkage of the system (Hosford, 2006). Considering the relationship between the diffusion and stress state Coble (1958), Johnson (1969) and De Jonghe \& Rahaman (1988) formulated mathematical models for sintering force between two particles. The discrete element method provided a suitable framework for more general application of these models (Parhami and McMeeking, 1998; Martin et al., 2006).

In our model, the particle interaction during sintering is described by the equation derived by Parhami and McMeeking (1998):

$$
F_{n}=\frac{\pi a^{4}}{8 D_{b}} v_{n}-\pi \gamma_{s}\left[4 R\left(1-\cos \frac{\Psi}{2}\right)+\operatorname{asin} \frac{\Psi}{2}\right]
$$

where $F_{n}$ is the normal force between two particles, $v_{n}$ - the normal relative velocity, $R$ - the particle radius, $a$ - the sintering contact radius, $\Psi$ - the dihedral angle, $\gamma_{s}$ - the surface energy and $D_{b}$ - the effective grain boundary diffusion coefficient. The geometric parameters used in Eq. (10) are defined in Fig. 7. In our model the translational motion will be considered only, assuming that the rotational motion is negligible in sintering. The tangential component is assumed as small in comparison with the normal one and is neglected. 


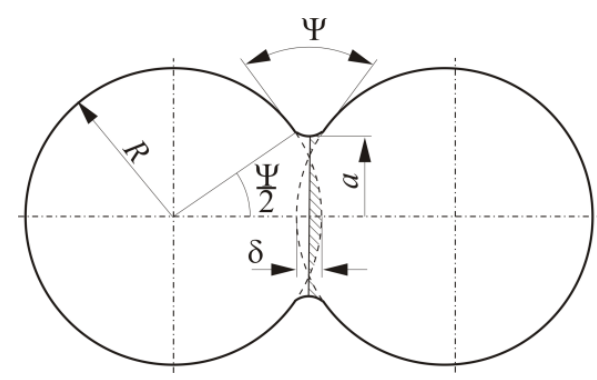

Fig. 7. Model parameters definition

The effective diffusion coefficient is given by the following equation, cf. (Parhami and McMeeking, 1998):

$D_{b}=\frac{D_{g} \delta_{g} \Omega}{k T}$

where $D_{g}$ - diffusion coefficient, $\delta_{g}$ - thickness of the grain boundary, $\Omega$ - atomic volume, $k$ - Boltzmann constant, $T$ sintering temperature. The initial neck radius $a_{0}$ depends on the initial penetration $u_{0}$ induced by the compaction. From simple geometrical considerations we have:

$$
a_{0}=\sqrt{\frac{R u_{n 0}}{2}}
$$

The growth of the radius of the interparticle grain boundary is governed by the following evolution law:

$\dot{a}=-\frac{R v_{n}}{a}$

The grain boundary radius a grows until the sintering process is stopped. Its maximum at the equilibrium state is given by the following geometric relationship:

$a_{\text {max }}=R \sin \frac{\Psi}{2}$

where: $\Psi$ is the dihedral angle determined according to the Young's law which assumes that in the system consisting of two grains and a gas in the pore in the equilibrium we have equlibrium of surface tensions.

The model described by Eq. (10) has been derived for identical particles. Following (Martin et al., 2006) it can be generalized for different size particles by replacing the radius $R$ in Eqs. (10)(13) with the equivalent particle radius $\bar{R}$ given by the following formula:

$\bar{R}=-\frac{2 R_{i} R_{j}}{R_{i}+R_{j}}$

On the right-hand side of Eq. (10) we have two terms, the first term has a character of viscous resistance to the particle approaching caused by the sintering attracting force represented by the second term. Denoting these force components, $F^{v}$ and $F^{\sin t}$, respectively, Eq. (10) can be rewritten as follows:

$F_{n}=F^{v}+F^{\text {sint }}$

where:

$F^{v}=\frac{\pi a^{4}}{8 D_{b}} v_{n}$

$F^{\sin t}=-\pi \gamma_{s}\left[4 R\left(1-\cos \frac{\Psi}{2}\right)+\operatorname{asin} \frac{\Psi}{2}\right]$

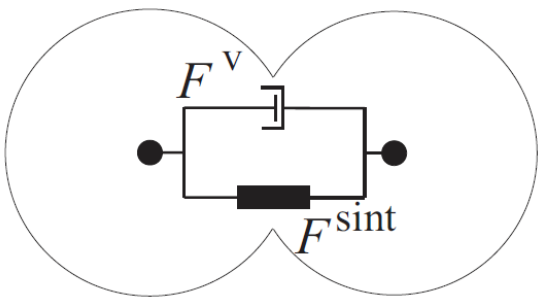

Fig. 8. Rheological scheme of the sintering model

The decomposition (15) of the interaction force in the sintering model can be represented by the rheological scheme shown in Fig. 8.

\section{SIMULATION OF SINTERING OF A CYLINDRICAL SPECIMEN}

The model implementation has been validated using the results of laboratory tests of sintering of NiAl powder presented in Sec. 2 Maintaining the original grain size (particle mean diameter $25 \mu \mathrm{m}$ ), a cylindrical container of diameter $200 \mu \mathrm{m}$ has been filled with 350 particles (Fig. 9a). It has been assumed that such a reduced geometric model represents correctly sintering process in a real specimen with diameter of $120 \mathrm{~mm}$. This assumption is justified provided the parameters characterizing sintering are uniformly distributed in a real specimen volume.

The particles in equilibrium under gravity (Fig. 9a) have been subjected to linearly rising compressive pressure of the rigid punch until the final load of $30 \mathrm{MPa}$ has been achieved. Then the increase of temperature to $1400{ }^{\circ} \mathrm{C}$ has been prescribed and the sintering process has been activated. The sintering has been treated as an isothermal process. The final configuration of the particles after sintering for 30 mins is shown in Fig. 9b. A significant reduction of the specimen height due to macroscopic shrinkage can be clearly observed.

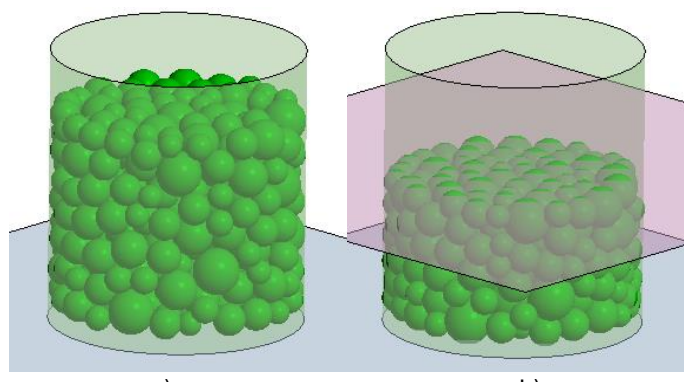

a)

b)

Fig. 9. Simulation of sintering: a) initial configuration,

b) final configuration

The model parameters are given in Tab. 2. In the calibration procedure, the effective diffusion coefficient, has been treated as a fitting parameter similarly as in Wonisch et al., (2009). The other parameters have been kept constant during calibration. Their values have been estimated based on literature data.

In order to increase numerical efficiency of the simulation based on the explicit time integration mass scaling has been employed. Mass scaling is typically used in the explicit time integration to increase a critical time step value. Since the 
process is very slow, it is possible to increase algorithmically inertial terms without affecting much the whole solution.

The calibration procedure has been based on fitting the density evolution to experimental results The relative density evolution obtained in the numerical simulation shown in Fig. 10 agrees well with experimental measurements which demonstrates a good performance of the numerical model.

Tab. 2. Material data and model parameters for NiAl sintering

\begin{tabular}{|l|c|}
\hline \multicolumn{1}{|c|}{ Parameter name } & Parameter value \\
\hline Density, & $5.91 \mathrm{~g} / \mathrm{cm}^{3}$ \\
\hline Contact stiffness, $k_{n}$ and $k_{t}$ & $7 \cdot 10^{5} \mathrm{~N} / \mathrm{m}$ \\
\hline Damping coefficient, $\xi$ & 0.7 \\
\hline Friction coefficient, $\mu$ & 0.05 \\
\hline Diffusion coefficient, $D_{g} \delta_{g}$ & $1.8 \cdot 10^{-19} \mathrm{~m}^{3} / \mathrm{s}$ \\
\hline Atomic volume, $\Omega$ & $1.21 \cdot 10^{-29} \mathrm{~m}^{3}$ \\
\hline Surface energy, $\gamma_{s}$ & $1.58 \mathrm{~J} / \mathrm{m}^{2}$ \\
\hline Dihedral angle, $\Psi$ & 1500 \\
\hline
\end{tabular}

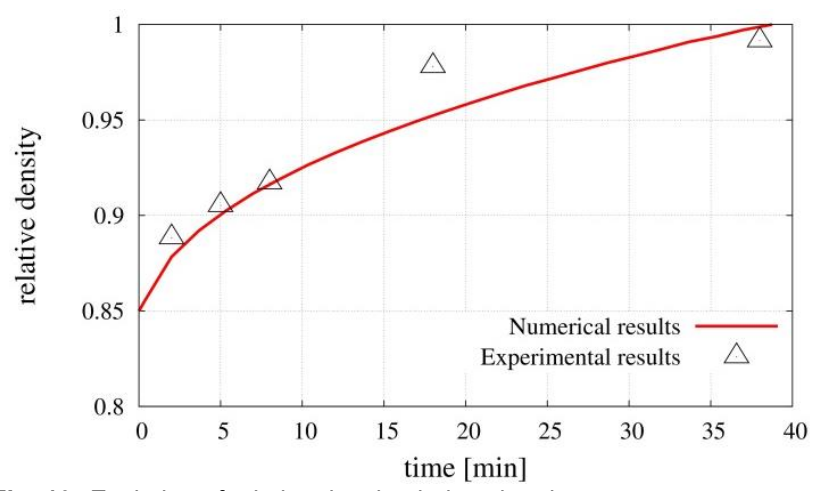

Fig. 10. Evolution of relative density during sintering process

\section{CONCLUDING REMARKS}

The results presented in the paper show a big potential of the presented numerical model in simulation of powder sintering. The discrete element method is a suitable tool to model powder sintering. The model reproduces correctly mechanism of sintering under pressure (shrinkage, change of density). The evolution of the density has been studied in this paper, however, the model can also be used to investigate other phenomena of a sintering process such as stresses during and after process. Further work on extension of the model is ongoing.

\section{REFERENCES}

1. Abouaf M., Chenot J.L., Raisson G., Bauduin P. (1988), Finite element simulation of hot isostatic pressing of metal powders, International Journal for Numerical Methods in Engineering, 25, 191-212.

2. Coble R.L. (1958), Initial Sintering of Alumina and Hematite, J. Amer. Ceramic Soc., 41, 55-62.

3. Cocks A.C.F. (1989), Inelastic deformation of porous materials, Journal of the Mechanics and Physics of Solids, 37 (6), 693-715.

4. De Jonghe L.C., Rahaman M.N. (1988), Sintering Stress of Homogeneous and Heterogeneous Powder Compacts, Acta Metall., 36, 223-229.
5. Duva J.M., Crow P.D. (1992), The densification of powders by power-law creep during hot isostatic pressing, Acta Metallurgica et Materialia, 40, 31-35.

6. Henrich B. (2007), (PhD thesis) Partikelbasierte Simulationsmethoden in Pulvertechnologie und Nanofluidik, Albert-LudwigsUniversität Freiburg im Breisgau.

7. Henrich B., Wonisch A., Kraft T., Moseler M., Riedel H. (2007), Simulations of the influence of rearrangement during sintering, Acta Materialia, 55, 753-762.

8. Hosford W.F. (2006), Material Science, Cambridge University Press.

9. Huilong Z., Averback R.S. (1996), Sintering processes of two nanoparticles: a study by molecular-dynamics simulations, Phil. Mag. Let., 73(1), 27-33.

10. Johnson D.L. (1969), New Method of Obtaining Volume, Grain Boundary, and Surface Diffusion Coefficients from Sintering Data, Journal of Applied Physics, 40, 192-200.

11. Kadau K., Entel P., Lomdahl P.S. (2002), Molecular-dynamics study of martensitic transformations in sintered Fe-Ni nanoparticles, Computer Physics Communications, 147, 126-129.

12. Kadushnikov R.M., Skorokhod V.V., Kamenin I.G., Alievskii V.M., Yu Nurkanov E., Alievskii D.M. (2001), Theory and technology of sintering, heat, and chemical heat-treatment processes computer simulation of spherical particle sintering. Powder Metallurgy and Metal Ceramics, 40(3-4), 154-163.

13. Luding S., Manetsberger K., Müllers J. (2005), A discrete model for long time sintering, Journal of Mechanics and Physics of Solids, 53, 455-491

14. Martin C.L., Schneider L.C.R., Olmos L., Bouvard D. (2006), Discrete element modeling of metallic powder sintering, Scripta Materialia, 55, 425-428.

15. Matsubara H. (1999), Computer simulations for the design of microstructural developments in ceramics, Computational Materials Science, 14, 125-128.

16. Olmos L., Martin C.L., Bouvard D. (2009), Sintering of mixtures of powders: experiments and modelling, Powder Technology, 190, 134-140.

17. Parhami F., McMeeking R.M. (1998), A network model for initial stage sintering,Mechanics of Materials, 27, 111-124.

18. Ponte Castañeda P. (1991), The effective mechanical properties of nonlinear isotropic composites, Journal of the Mechanics and Physics of Solids, 39, 45-71.

19. Rojek J., Pietrzak K., Chmielewski M., Kaliński D., Nosewicz S. (2011), Discrete Element Simulation of Powder Sintering, Computer Methods in Materials Science, 11, 68-73.

20. Sofronis P., McMeeking R.M. (1992), Creep of power-law materia containing spherical voids, ASME Journal of Applied Mechanics, 59, S88-S95.

21. Wonisch A., Kraft T., Moseler M., Riedel H. (2009), Effect of different particle size distributions on solid-state sintering: A microscopic simulation approach, J. Am. Ceram. Soc., 92, 14281434.

22. Zachariah M.R., Carrier M.J. (1999), Molecular dynamics computation of gas-phase nanoparticle sintering: a comparison with phenomenological models, Journal of Aerosol Science, 30, 11391151.

23. Zeng P., Zajac S., Clapp P.C., Rifkin J.A. (1998), Nanoparticle sintering simulations, Materials Science and Engineering, A252, 301-306.

24. Zhu H., Averback R.S. (1995), Molecular dynamics simulations of densification process in nanocrystalline materials, Materials Science and Engineering A, A204(1-2), 96-100.

Acknowledgements: The results presented in this paper have been obtained within the project "KomCerMet" (contract no. POIG.01.03.0114-013/08-00 with the Polish Ministry of Science and Higher Education) in the framework of the Operational Programme Innovative Economy 2007-2013. 